\title{
Analytic functions in the unit ball of bounded $L$-index in joint variables and of bounded $L$-index in direction: a connection between these classes
}

https://doi.org/10.1515/dema-2019-0008

Received September 16, 2018; accepted January 14, 2019

Abstract: We give negative answer to the question of Bordulyak and Sheremeta for more general classes of entire functions than in the original formulation: Does index boundedness in joint variables for an entire function $F$ imply index boundedness in the variable $z_{j}$ for the function $F$ ? This question is addressed for entire functions of bounded $\mathbf{L}$-index in joint variables and entire functions of bounded $L$-index in direction. We also present a class of analytic functions in the unit ball which has bounded $\mathbf{L}$-index in joint variables and has unbounded $l$-index in the variables $z_{1}$ and $z_{2}$ for any positive continuous function $l: \mathbb{B}^{2} \rightarrow \mathbb{C}$.

Keywords: bounded $L$-index in direction, entire function of several variables, bounded $\mathbf{L}$-index in joint variables, analytic function in the unit ball, existence theorem

MSC: 30D15, 30D30, 34M05

\section{Introduction}

For $K=\left(k_{1}, \ldots, k_{n}\right) \in \mathbb{Z}_{+}^{n}$ let us denote $\|K\|=k_{1}+\cdots+k_{n}, K !=k_{1} ! \cdot \ldots \cdot k_{n} !$. For $A=\left(a_{1}, \ldots, a_{n}\right) \in$ $\mathbb{C}^{n}, B=\left(b_{1}, \ldots, b_{n}\right) \in \mathbb{C}^{n}$, we will use the formal notations without violation of the existence of these expressions $A \pm B=\left(a_{1} \pm b_{1}, \ldots, a_{n} \pm b_{n}\right), A B=\left(a_{1} b_{1}, \cdots, a_{n} b_{n}\right), A^{B}=a_{1}^{b_{1}} a_{2}^{b_{2}} \cdot \ldots a_{n}^{b_{n}}$. For a partial derivative of entire function $F(z)=F\left(z_{1}, \ldots, z_{n}\right)$ we will use the notation $F^{(K)}(z)=\frac{\partial^{\|K\|} F}{\partial z^{K}}=\frac{\partial^{k_{1}+\cdots+k_{n}} F}{\partial z_{1}^{k_{1}} \ldots \partial z_{n}^{k_{n}}}$, where $K=\left(k_{1}, \ldots, k_{n}\right) \in \mathbb{Z}_{+}^{n}$.

Let $\mathbf{L}(z)=\left(l_{1}(z), \ldots, l_{n}(z)\right)$, where $l_{j}(z)$ are positive continuous functions of variable $z \in \mathbb{C}^{n}, j \in$ $\{1,2, \ldots, n\}$.

An entire function $F(z)$ is called a function of bounded L-index in joint variables [1, 2], if there exists a number $m \in \mathbb{Z}_{+}$such that for each $J=\left(j_{1}, j_{2}, \ldots, j_{n}\right) \in \mathbb{Z}_{+}^{n}$ and for all $z \in \mathbb{C}^{n}$

$$
\frac{\left|F^{(J)}(z)\right|}{J ! \mathbf{L}^{J}(z)} \leq \max \left\{\frac{\left|F^{(K)}(z)\right|}{K ! \mathbf{L}^{K}(z)}: K \in \mathbb{Z}_{+}^{n},\|K\| \leq m\right\} .
$$

The least such integer $m$ for which inequality (1) holds is called the $\mathbf{L}$-index in joint variables of the function $F$ and is denoted by $N(F, \mathbf{L})$. Besides, by $N\left(F, z^{0}, \mathbf{L}\right)$ we denote the $\mathbf{L}$-index in joint variables of the function $F$ at the point $z^{0}$, i.e. it is the least integer $m$ for which inequality (1) holds for every $J$ with $z^{0}$ instead of $z$.

\footnotetext{
*Corresponding Author: Andriy Bandura: Department of Advanced Mathematics, Ivano-Frankivsk National Technical University of Oil and Gas, Ukraine, Postal address: 15 Karpatska street, Ivano-Frankivsk, 76019, Ukraine; E-mail: andriykopanytsia@gmail.com Oleh Skaskiv: Department of Mathematics and Mechanics, Ivan Franko National University of L'viv, Ukraine, Postal address: 1 Universytetska street, Lviv, 79000, Ukraine; E-mail: olskask@gmail.com
} 
If $l_{j}\left(z_{j}\right) \equiv 1, j \in\{1,2, \ldots, n\}$, then the entire function is called a function of bounded index in joint variables [3-5]. If $n=1$ then we get the definition [6] of bounded $l$-index for an entire function in the complex plane. And if in addition $l \equiv 1$ then we obtain the definition [7] of entire function of bounded index.

The polydisc $\left\{z \in \mathbb{C}^{n}:\left|z_{j}-z_{j}^{0}\right|<r_{j}, j \in\{1, \ldots, n\}\right\}$ is denoted by $\mathbb{D}^{n}\left(z^{0}, R\right)$, its skeleton $\left\{z \in \mathbb{C}^{n}:\right.$ $\left.\left|z_{j}-z_{j}^{0}\right|=r_{j}, j \in\{1, \ldots, n\}\right\}$ is denoted by $T^{n}\left(z^{0}, R\right)$. For $R \in \mathbb{R}_{+}^{n}, j \in\{1, \ldots, n\}$ and $\mathbf{L}(z)=\left(l_{1}(z), \ldots, l_{n}(z)\right)$ we define

$\lambda_{1, j}(R)=\inf _{z^{0} \in \mathbb{C}^{n}} \inf \left\{l_{j}(z) / l_{j}\left(z^{0}\right): z \in \mathbb{D}^{n}\left[z^{0}, R / \mathbf{L}\left(z^{0}\right)\right]\right\}, \lambda_{2, j}(R)=\sup _{z^{0} \in \mathbb{C}^{n}} \sup \left\{l_{j}(z) / l_{j}\left(z^{0}\right): z \in \mathbb{D}^{n}\left[z^{0}, R / \mathbf{L}\left(z^{0}\right)\right]\right\}$.

By $Q^{n}$ we denote a class of functions $\mathbf{L}(z)$ which for every $R \in \mathbb{R}_{+}^{n}$ satisfy the condition $0<\lambda_{1, j}(R) \leq$ $\lambda_{2, j}(R)<+\infty$.

A concept of entire function of bounded $\mathbf{L}$-index in joint variables was introduced by Bordulyak and Sheremeta [8] for the function $\mathbf{L}(z)$ of special form. They considered a case $\mathbf{L}(z)=\left(l_{1}\left(\left|z_{1}\right|\right), \ldots, l_{n}\left(\left|z_{n}\right|\right)\right)$, where every $l_{j}$ is a positive continuous function. In this paper we reference Bordulyak-Sheremeta $\mathbf{L}$-index as the "BS L-index". They formulated some criteria of BS L-index boundedness and some properties of these functions without proofs. Among those properties we would like to mention the following:

Proposition 1. [8] Let $\mathbf{L} \in Q^{n}$ and $\mathbf{L}(z)=\left(l_{1}\left(\left|z_{1}\right|\right), \ldots, l_{n}\left(\left|z_{n}\right|\right)\right)$. If an entire function $F: \mathbb{C}^{n} \rightarrow \mathbb{C}$ is of bounded $B S$ L-index then

$$
\ln \max \left\{|F(z)|:\left|z_{j}\right|=r_{j}, j \in\{1, \ldots, n\}\right\}=O\left(l_{1}\left(r_{1}\right)+\ldots+l_{n}\left(r_{n}\right)\right)
$$

as $r_{1}+\ldots+r_{n} \rightarrow \infty$.

Bordulyak and Sheremeta [8] presented an example of entire function $F(z)=\exp \left\{z_{1} \cdots z_{n}\right\}$. The function has bounded index in every variable for fixed others, but its magnitude as a function of other variables is unbounded. Taking into account Proposition 1 it is unbounded BS $L$-index for any $\mathbf{L}(z)=\left(l_{1}\left(\left|z_{1}\right|\right), \ldots, l_{n}\left(\left|z_{n}\right|\right)\right)$. Particularly, index boundedness in each variable does not imply index boundedness in joint variables.

Recently, a more general vector-function $\mathbf{L}(z)=\left(l_{1}(z), \ldots, l_{n}(z)\right)$ was proposed [9], where every $l_{j}: \mathbb{C}^{n} \rightarrow$ $\mathbb{R}_{+}$is a positive continuous function, $j \in\{1, \ldots, n\}$. For such a function $\mathbf{L}: \mathbb{C}^{n} \rightarrow \mathbb{R}_{+}^{n}$ and an entire function $F: \mathbb{C}^{n} \rightarrow \mathbb{C}$ there was also considered a concept of $\mathbf{L}$-index boundedness in joint variables. A definition was provided at the beginning of the Introduction section. All propositions from [8] are presented with full proofs in $[9,10]$ for the more general function $\mathbf{L}$. Also, it was proved [9] that if an entire function $F: \mathbb{C}^{n} \rightarrow \mathbb{C}$ has uniformly bounded index in every variable $z_{j}$ then the function $F$ is of bounded index in joint variables. Note that the bounded index in the variable $z_{j}$ is equivalent to bounded index in the direction $\mathbf{1}_{j}=(0, \ldots, 0, \underbrace{1}, 0, \ldots, 0)$ (see definition and properties in $\left.[9,11]\right)$.

$j$-th place

Bordulyak and Sheremeta wrote [8]: "we could not establish whether the index boundedness in joint variables implies the index boundedness in each variable".

In this paper, we give an answer to the question in more general case: "Does L-index boundedness in joint variables for an entire function $F$ imply $l_{j}$-index boundedness in the variable $z_{j}$ for the function $F$ and for every $j \in\{1, \ldots, n\} ?^{\prime \prime}$

We also consider the similar problem for analytic functions in the unit ball.

\section{Entire functions}

Let $F$ be an entire function and $\mathbb{Z}_{F}$ be a zero set of the $F$. If $z^{0} \in Z_{F}$, then we denote by $p_{F}\left(z^{0}\right)$ the multiplicity of the zero point $z^{0}$ of the function $F$, i.e. for all $J,\|J\|<p_{F}\left(z^{0}\right), F^{(J)}\left(z^{0}\right)=0$, but at least one $J,\|J\|=p_{F}\left(z^{0}\right)$, one has $F^{(J)}\left(z^{0}\right) \neq 0$.

We need the following theorem: 
Theorem 1. [12] In order that for an entire function $F$ there exists a positive continuous function $\mathbf{L}(z)=$ $\left(l_{1}(z), \ldots, l_{n}(z)\right)$ such that $F(z)$ is a function of bounded $\mathbf{L}$-index in joint variables it is necessary and sufficient that there exists $p \in \mathbb{Z}_{+}$such that $p_{F}\left(z^{0}\right) \leq p$ for all $z^{0} \in Z_{F}$.

Using Theorem 1 we prove the following theorem about class of entire functions of unbounded $l$-index in the variable $z_{1}$ and in the variable $z_{2}$ for any positive continuous function $l: \mathbb{C}^{2} \rightarrow \mathbb{R}_{+}$.

Theorem 2. Let $f: \mathbb{C} \rightarrow \mathbb{C}$ be an entire function with unbounded multiplicities of zeros. Then the function $F\left(z_{1}, z_{2}\right)=z_{1} z_{2}+z_{1} f\left(z_{1}\right)+z_{2} f\left(z_{2}\right)$ is of bounded $\mathbf{L}$-index in joint variables for some positive continuous vectorfunction $\mathbf{L}: \mathbb{C}^{2} \rightarrow \mathbb{R}_{+}^{2}$ and $F\left(z_{1}, z_{2}\right)$ is of unbounded l-index in the variable $z_{1}$ and in the variable $z_{2}$ for any positive continuous function $l: \mathbb{C}^{2} \rightarrow \mathbb{R}_{+}$.

Proof. Let $z^{0}$ be an arbitrary zero point of the function $F$. Since $\frac{\partial^{2} F}{\partial z_{1} \partial z_{2}}=1 \neq 0$ it means that the multiplicity $p_{F}\left(z^{0}\right)$ of zero points of the function $F$ is not greater than 2 . Then by Theorem 1 the function $F\left(z_{1}, z_{2}\right)=$ $z_{1} z_{2}+z_{1} f\left(z_{1}\right)+z_{2} f\left(z_{2}\right)$ has bounded $\mathbf{L}$-index in joint variables for some positive continuous vector-function $\mathbf{L}: \mathbb{C}^{2} \rightarrow \mathbb{R}_{+}^{2}$

Put $z_{2}=0$. Then $F\left(z_{1}, 0\right)=z_{1} f\left(z_{1}\right)$. The function has unbounded multiplicity of zeros because $f$ is a function with unbounded multiplicities of zeros. Denote $g(t)=t f(t)$. Let $t_{p}$ be a zero of the $g(t)$ with multiplicity $p$. Hence, we have $g^{(p)}\left(t_{p}\right) \neq 0$ and $g^{(j)}\left(t_{p}\right)=0$ for all $j \leq p-1$. Therefore, the $l$-index at the point $t_{p}$ is not less than $p$, i.e.

$$
N\left(g, l, z^{0}\right) \geq p
$$

for any positive continuous function $l: \mathbb{C}^{2} \rightarrow \mathbb{R}_{+}$. If $p \rightarrow+\infty$, then $N\left(F, \mathbf{L}, z^{0}\right) \rightarrow+\infty$. Thus, $g(t)$ is of unbounded $l$-index. Therefore, $F\left(z_{1}, z_{2}\right)$ has unbounded $l$-index in the variable $z_{1}$. Of course, the similar statement is also true in the variable $z_{2}$.

Theorem 3. Let $\Phi(t)=\prod_{j=1}^{\infty}\left(1+t 2^{-j}\right)^{j}$. Then the entire function $F\left(z_{1}, z_{2}\right)=z_{1}+\Phi\left(z_{2}\right)$ has

1) bounded $\mathbf{L}$-index in joint variables $N(F, \mathbf{L})=1$ with $\mathbf{L}\left(z_{1}, z_{2}\right)=\left(1, C \exp \left\{\left(\left|z_{2}\right|+1\right) / 2\right\}\right)$, C is a some constant (i.e. in a sense of Bordulyak-Sheremeta);

2) unbounded l-index in the variable $z_{2}$ for any positive continuous function $l: \mathbb{C}^{2} \rightarrow \mathbb{R}_{+}$.

Proof. For arbitrary $\left(z_{1}, z_{2}\right) \in \mathbb{C}^{2}$ we put $r_{j}=\left|z_{j}\right|, j \in\{1,2\}$. Since $\frac{\partial F}{\partial z_{1}} \equiv 1$ this yields

$$
\max \left\{\frac{\left|F^{\left(j_{1}, j_{2}\right)}\left(z_{1}, z_{2}\right)\right|}{j_{1} ! j_{2} !}: j_{1}+j_{2} \leq 1\right\} \geq 1 .
$$

For any $j_{2} \in \mathbb{Z}$ and $j_{1} \geq 2 F^{\left(j_{1}, j_{2}\right)}\left(z_{1}, z_{2}\right) \equiv 0$. For $j_{1}=1$ and for any $j_{2} \geq 1$ we have $F^{\left(1, j_{2}\right)}\left(z_{1}, z_{2}\right) \equiv 0$. Let $j_{1}=0$. Using Cauchy's formula in the variable $z_{2}$ we deduce that for all $j_{2} \geq 2$

$$
\begin{aligned}
\frac{\left|F^{\left(0, j_{2}\right)}\left(z_{1}, z_{2}\right)\right|}{j_{2} !} & =\left|\frac{1}{2 \pi i} \int_{\left|\tau_{2}-z_{2}\right|=1} \frac{F^{(0,1)}\left(z_{1}, \tau_{2}\right)}{\left(\tau_{2}-z_{2}\right)^{j_{2}}} d \tau_{2}\right| \\
& \leq \max \left\{\left|F^{(0,1)}\left(z_{1}, \tau_{2}\right)\right|:\left|\tau_{2}\right| \leq r_{2}+1\right\} \\
& =\max \left\{\left|\Phi^{\prime}\left(\tau_{2}\right)\right|:\left|\tau_{2}\right|=r_{2}+1\right\},
\end{aligned}
$$

where $r_{2}=\left|z_{2}\right|$.

In [13] for the function $\Phi$ there was obtained

$$
\frac{\left|\Phi^{(p)}(t)\right|}{p !} \leq C \exp \{|t| / 2\} \text { for any } p \in \mathbb{N},
$$

where $C$ is a some positive constant. Thus, inequalities (3) and (4) yield

$$
\frac{\left|F^{\left(0, j_{2}\right)}\left(z_{1}, z_{2}\right)\right|}{j_{2} !} \leq C \exp \left\{\left(r_{2}+1\right) / 2\right\} \text { for } j_{2} \geq 2 \text {. }
$$


A positive continuous vector-function $\mathbf{L}(z)=\left(l_{1}(z), l_{2}(z)\right)$ can be chosen such

$$
l_{1}\left(z_{1}\right) \equiv 1, l_{2}\left(z_{2}\right):=C \exp \left\{\left(\left|z_{2}\right|+1\right) / 2\right\}
$$

Therefore,

$$
\max \left\{\frac{\left|F^{\left(k_{1}, k_{2}\right)}\left(z_{1}, z_{2}\right)\right|}{k_{1} ! k_{2} ! l_{1}^{k_{1}}\left(z_{1}\right) l_{2}^{k_{2}}\left(z_{2}\right)}: k_{1}+k_{2} \leq 1\right\}=\max \left\{\left|F\left(z_{1}, z_{2}\right)\right|, 1, \frac{\left|\Phi^{\prime}\left(z_{2}\right)\right|}{l_{2}\left(z_{2}\right)}\right\} \geq 1 .
$$

Note that $F^{(1,1)} \equiv 0$. From (3), (4), (5), (6) it follows that for all $j_{1}+j_{2} \geq 2$

$$
\frac{\left|F^{\left(j_{1}, j_{2}\right)}\left(z_{1}, z_{2}\right)\right| /\left(j_{1} ! j_{2} ! l_{1}^{j_{1}}\left(z_{1}\right) l_{2}^{j_{2}}\left(z_{2}\right)\right)}{\max \left\{\frac{\left|F^{\left(k_{1}, k_{2}\right)}\left(z_{1}, z_{2}\right)\right|}{k_{1} ! k_{2} ! l_{1}^{k_{1}}\left(z_{1}\right) l_{2}^{k_{2}}\left(z_{2}\right)}: k_{1}+k_{2} \leq 1\right\}} \leq \frac{\max \left\{1,\left|\Phi^{\prime}\left(\tau_{2}\right)\right|:\left|\tau_{2}\right|=r_{2}+1\right\}}{j_{2} ! l_{2}^{j_{2}}\left(z_{2}\right)} \leq 1 .
$$

Hence, we have

$$
\frac{\left|F^{\left(j_{1}, j_{2}\right)}\left(z_{1}, z_{2}\right)\right|}{j_{1} ! j_{2} ! l_{1}^{j_{1}}\left(z_{1}\right) l_{2}^{j_{2}}\left(z_{2}\right)} \leq \max \left\{\frac{\left|F^{\left(k_{1}, k_{2}\right)}\left(z_{1}, z_{2}\right)\right|}{k_{1} ! k_{2} ! l_{1}^{k_{1}}\left(z_{1}\right) l_{2}^{k_{2}}\left(z_{2}\right)}: k_{1}+k_{2} \leq 1\right\} .
$$

It should be observed that $\frac{\left|F^{(1,0)}\left(z_{1}, z_{2}\right)\right|}{1 ! l_{1}\left(z_{1}\right)}=1>0=\left|F\left(z_{1}, z_{2}\right)\right|=\left|F^{(0,1)}\left(z_{1}, z_{2}\right)\right|$ for $\left(z_{1}, z_{2}\right) \in Z_{F}$ and $\frac{\left|F^{(1,0)}\left(z_{1}, z_{2}\right)\right|}{1 ! l_{1}\left(z_{1}\right)}=1>0=\left|F^{(0,1)}\left(z_{1}, z_{2}\right)\right|$ for $\left(z_{1}, z_{2}\right) \in Z_{F^{(0,1)}}$. Therefore, $N(F, \mathbf{L})=1$.

The second part of the theorem can be proved by analogy to the proof of Theorem 2 .

Actually, we showed in Theorems 2-3 that bounded L-index in joint variables for entire function does not provide bounded $l_{j}$-index in the direction $\mathbf{1}_{j}$.

\section{Analytic functions in the unit ball}

Here we will consider similar problems for analytic functions in the unit ball.

All notations from previous sections remain valid. However, we need additional notations. The open ball $\left\{z \in \mathbb{C}^{n}:\left|z-z^{0}\right|<r\right\}$ is denoted by $\mathbb{B}^{n}\left(z^{0}, r\right)$, the closed ball $\left\{z \in \mathbb{C}^{n}:\left|z-z^{0}\right| \leq r\right\}$ is denoted by $\mathbb{B}^{n}\left[z^{0}, r\right]$, $\mathbb{B}^{n}=\mathbb{B}^{n}(\mathbf{0}, 1), \mathbb{D}=\mathbb{B}^{1}=\{z \in \mathbb{C}:|z|<1\}$, where $|z|=\sqrt{\sum_{j=1}^{n}\left|z_{j}\right|^{2}}, \mathbf{0}=(0, \ldots, 0), \mathbf{1}=(1, \ldots, 1) \in \mathbb{R}_{+}^{n}$. Let $\mathbf{L}(z)=\left(l_{1}(z), \ldots, l_{n}(z)\right)$, where $l_{j}(z): \mathbb{B}^{n} \rightarrow \mathbb{R}_{+}$is a continuous function such that

$$
\left(\forall z \in \mathbb{B}^{n}\right): l_{j}(z)>\beta /(1-|z|), j \in\{1, \ldots, n\},
$$

where $\beta>\sqrt{n}$ is some constant.

An analytic function $F: \mathbb{B}^{n} \rightarrow \mathbb{C}$ is said to be of bounded $\mathbf{L}$-index (in joint variables) [14, 15], if there exists $n_{0} \in \mathbb{Z}_{+}$such that for all $z \in \mathbb{B}^{n}$ and for all $J \in \mathbb{Z}_{+}^{n}$ inequality (1) holds.

Above we used Theorem 1 for entire functions. The theorem states that for every entire function $F: \mathbb{C}^{n} \rightarrow$ $\mathbb{C}$ with bounded multiplicities of zero points there exists a positive continuous vector-function $\mathbf{L}: \mathbb{C}^{n} \rightarrow \mathbb{R}_{+}^{n}$ such that $F$ has bounded L-index in joint variables.

Here we consider a similar problem for analytic functions in the unit ball: Is there a positive continuous vector function $\mathbf{L}: \mathbb{B}^{n} \rightarrow \mathbb{R}_{+}^{n}$ for any analytic function $F: \mathbb{B}^{n} \rightarrow \mathbb{C}$ with bounded multiplicities of zeros such that $F$ has bounded $\mathbf{L}$-index in joint variables?

In this paper, we give a positive answer to the question.

Theorem 4. In order that for an analytic function $F: \mathbb{B}^{n} \rightarrow \mathbb{C}$ there exist a positive continuous function $\mathbf{L}(z)=$ $\left(l_{1}(z), \ldots, l_{n}(z)\right)$ satisfying (7) such that $F$ is a function of bounded $\mathbf{L}$-index in joint variables it is necessary and sufficient that there exists $p \in \mathbb{Z}_{+}$such that $p_{F}\left(z^{0}\right) \leq p$ for all $z^{0} \in Z_{F}$. 
Proof. The proof is similar to the proof of Theorem 1 in [12] for entire functions.

Necessity. To simplify the notation we consider everywhere in the proof $p_{0}:=p_{F}\left(z^{0}\right)$. Necessity follows from the definition of bounded $\mathbf{L}$-index in joint variables. Indeed, assume on the contrary that

$$
\left(\forall p \in \mathbb{Z}_{+}\right)\left(\exists z^{0} \in Z_{F}\right): \quad p_{0}>p .
$$

This means that for some $J^{0} \in \mathbb{Z}_{n}^{+},\left\|J^{0}\right\|=p_{0}$, one has $F^{\left(J^{0}\right)}\left(z^{0}\right) \neq 0$ and $F^{(J)}\left(z^{0}\right)=0$ for all $J,\|J\| \leq p_{0}-1$ and $z^{0} \in Z_{F}$. Therefore, the $\mathbf{L}$-index in joint variables at the point $z^{0}$ is not less than $p_{0}>p$, i.e.

$$
N\left(F, \mathbf{L}, z^{0}\right) \geq p_{0}>p .
$$

If $p \rightarrow+\infty$, then we obtain that $N\left(F, \mathbf{L}, z^{0}\right) \rightarrow+\infty$. This contradicts the boundedness of $\mathbf{L}$-index in joint variables of the function $F$.

Sufficiency. Let $p$ be the least integer such that $\forall z^{0} \in Z_{F} p_{0} \leq p$. Denote

$$
\max \{A, B\}=\left(\max \left\{a_{1}, b_{1}\right\}, \ldots, \max \left\{a_{n}, b_{n}\right\}\right)
$$

for $A, B \in \mathbb{R}_{+}^{n}$. Let $R \in \mathbb{R}_{+}^{n}$ be such that $|R|<1$. We define $r_{0}=\frac{\min \{1-|R|,|R|\}}{2 \sqrt{n}}, K_{R}=\left\{z \in \mathbb{C}^{n}: z \in \mathbb{D}^{n}\left[\mathbf{0}, R+r_{0}\right.\right.$. 1] $\left.\backslash \mathbb{D}^{n}\left(\mathbf{0}, \max \left\{\mathbf{0}, R-r_{0} \cdot \mathbf{1}\right\}\right)\right\}$ for all $|R|<1$ and

$$
m_{1}(R)=\min _{z^{0} \in K_{R} \cap Z_{F}} \max _{\|J\| \leq p}\left\{\frac{\left|F^{(J)}\left(z^{0}\right)\right|}{J !}: F^{(J)}\left(z^{0}\right) \neq 0\right\} .
$$

Since $F$ is an analytic function, there exists $E=E(R)=\left(\varepsilon_{1}(R), \ldots, \varepsilon_{n}(R)\right)>\mathbf{0}$ such that

$$
\frac{\left|F^{\left(J^{0}\right)}(z)\right|}{J^{0 !}} \geq \frac{m_{1}(R)}{2}
$$

for all

$$
z \in K_{R} \cap G_{E}, \quad G_{E}=\bigcup_{z^{0} \in Z_{F}} \mathbb{D}^{n}\left(z^{0}, E(R)\right),
$$

and for some $J^{0},\left\|J^{0}\right\| \geq p_{0}$ with $F^{\left(J^{0}\right)}\left(z^{0}\right) \neq 0$.

Let us denote

$$
\begin{gathered}
m_{2}(R)=\min \left\{|F(z)|: z \in \mathbb{D}^{n}\left[\mathbf{0}, R+r_{0} \cdot \mathbf{1}\right], z \notin G_{E}\right\}, \\
Q(R)=\min \left\{m_{1}(R) / 2, m_{2}(R), 1\right\} .
\end{gathered}
$$

For every $z \in \mathbb{B}^{n}$ we put $R=\left(\left|z_{1}\right|, \ldots,\left|z_{n}\right|\right)$. Then at least one of the numbers $|F(z)|, \frac{\left|F^{()}(z)\right|}{J !}$ with $\|J\| \leq p$ is not less than $Q(R)$ (respectively, $\frac{\left|F^{\left(0^{0}\right)}(z)\right|}{J^{0} !}$ for $z \in K_{R} \cap G_{E}$ and $|F(z)|$ for $z \in K_{R} \backslash G_{E}$ ). This yields

$$
\max \left\{\frac{\left|F^{(J)}(z)\right|}{J !}:\|J\| \leq p\right\} \geq Q(R) .
$$

Using Cauchy's formula for polydisc $\mathbb{D}^{n}\left(z, r_{0} \cdot \mathbf{1}\right)$ we deduce that for all $J,\|J\| \geq p+1$

$$
\frac{\left|F^{(J)}(z)\right|}{J !}=\left|\frac{1}{(2 \pi i)^{n}} \int_{\mathbb{T}^{n}\left(z, r_{0} \cdot \mathbf{1}\right)} \frac{F(\tau)}{(\tau-z)^{J+1}} d \tau\right| \leq \frac{1}{r_{0}^{\|J\|}} \max \left\{|F(\tau)|: \tau \in \mathbb{D}^{n}\left(\mathbf{0}, R+r_{0} \cdot \mathbf{1}\right)\right\} .
$$

A positive continuous function $\mathbf{L}(z)=\left(l_{1}(z), \ldots, l_{n}(z)\right)$ can be chosen such that

$$
l_{1}(z)=\ldots=l_{n}(z):=\max \left\{\frac{\max \left\{1, \max \left\{|F(\tau)|: \tau \in \mathbb{D}^{n}\left(\mathbf{0}, R+r_{0} \cdot \mathbf{1}\right)\right\}\right\}}{Q(R) r_{0}^{2}}, \frac{\beta}{1-|R|}\right\},
$$

where $\beta>\sqrt{n}$ is some constant. 
From (8) and (3) it follows that for all $\|J\| \geq 2 p$

$$
\begin{aligned}
\frac{\left|F^{(J)}(z)\right| /\left(J ! \mathbf{L}^{J}(z)\right)}{\max \left\{\frac{\left|F^{(K)}(z)\right|}{K ! \mathbf{L}^{K}(z)}:\|K\| \leq p\right\}} & \frac{l_{1}^{-\|J\|}(z)}{Q(R) l_{1}^{-p}(z) r_{0}^{\|J\|}} \max \left\{|F(\tau)|: \tau \in \mathbb{D}^{n}\left(\mathbf{0}, R+r_{0} \cdot \mathbf{1}\right)\right\} \leq l_{1}^{p-\|J\|}(z) \times \\
& \times\left(\frac{\max \left\{1, \max \left\{|F(\tau)|: \tau \in \mathbb{D}^{n}\left(\mathbf{0}, R+r_{0} \cdot \mathbf{1}\right)\right\}\right\}}{Q(R) r_{0}^{2}}\right)\|J\| / 2 \\
& =l_{1}^{p-\|J\| / 2}(z) \leq 1 .
\end{aligned}
$$

Hence, we have

$$
\frac{\left|F^{(J)}(z)\right|}{J ! \mathbf{L}^{J}(z)} \leq \max \left\{\frac{\left|F^{(K)}(z)\right|}{K ! \mathbf{L}^{K}(z)}:\|K\| \leq 2 p\right\} .
$$

In view of arbitrariness of $z$, the analytic function $F$ has bounded $\mathbf{L}$-index in joint variables.

Using Theorem 4 it is possible to deduce the following corollary by analogy to the proof of Theorem 2 .

Corollary 1. Let $f: \mathbb{D} \rightarrow \mathbb{C}$ be an analytic function with unbounded multiplicities of zeros. Then the function $F\left(z_{1}, z_{2}\right)=z_{1} z_{2}+z_{1} f\left(z_{1}\right)+z_{2} f\left(z_{2}\right)$ is of bounded $\mathbf{L}$-index in joint variables for some positive continuous vectorfunction $\mathbf{L}: \mathbb{B}^{2} \rightarrow \mathbb{R}_{+}^{2}$ and $F\left(z_{1}, z_{2}\right)$ is of unbounded l-index in the variable $z_{1}$ and in the variable $z_{2}$ for any positive continuous function $l: \mathbb{B}^{2} \rightarrow \mathbb{R}_{+}$.

Acknowledgements: Authors are very thankful for the valuable comments and suggestions by anonymous reviewers.

\section{References}

[1] Bandura A., Skaskiv O., Entire functions of several variables of bounded index, Lviv, Publisher I. E. Chyzhykov, 2016

[2] Bandura A., Skaskiv 0., Entire functions of bounded L-index: Its zeros and behavior of partial logarithmic derivatives, J. Complex Analysis, 2017, Article ID 3253095, DOI: 10.1155/2017/3253095

[3] Nuray F., Patterson R. F., Entire bivariate functions of exponential type, Bull. Math. Sci., 2015, 5(2), 171-177, DOI: 10.1007/s13373-015-0066-x

[4] Nuray F., Patterson R. F., Multivalence of bivariate functions of bounded index, Le Matematiche, 2015, 70(2), 225-233, DOI: 10.4418/2015.70.2.14

[5] Patterson R. F., Nuray F., A characterization of holomorphic bivariate functions of bounded index, Math. Slov., 2017, 67(3), 731-736, DOI: $10.1515 / \mathrm{ms}-2017-0005$

[6] Kuzyk A. D., Sheremeta M. M., Entire functions of bounded l-distribution of values, Math. Notes., 1986, 39(1), 3-8, DOI:10.1007/BF01647624

[7] Lepson B., Differential equations of infinite order, hyperdirichlet series and entire functions of bounded index, Proc. Sympos. Pure Math., 1968, 2, 298-307

[8] Bordulyak M. T., Sheremeta M. M., Boundedness of the L-index of an entire function of several variables, Dopov. Akad. Nauk Ukr., 1993, 9, 10-13 (in Ukrainian)

[9] Bandura A. I., Bordulyak M. T., Skaskiv O. B., Sufficient conditions of boundedness of L-index in joint variables, Mat. Stud., 2016, 45(1), 12-26, DOI: 10.15330/ms.45.1.12-26

[10] Bandura A., Skaskiv O., Asymptotic estimates of entire functions of bounded L-index in joint variables, Novi Sad J. Math., 2018, 48(1), 103-116, DOI: 10.30755/NSJOM.06997

[11] Bandura A. I., Skaskiv O. B., Directional logarithmic derivative and the distribution of zeros of an entire function of bounded $L$-index along the direction, Ukrain. Mat. J., 2017, 69(3), 500-508, DOI: 10.1007/s11253-017-1377-8

[12] Bandura A. I., Skaskiv O. B., Iyer's metric space, existence theorem and entire functions of bounded $\mathbf{L}$-index in joint variables, Bukovyn. Mat. Zh., 2017, 5(3-4), 8-14 (in Ukrainian)

[13] Pugh W. J., Sums of functions of bounded index, Proc. Amer. Math. Soc., 1969, 22, 319-323

[14] Bandura A., Skaskiv O., Functions analytic in a unit ball of bounded L-index in joint variables, J. Math. Sci., 2017, 227(1), 1-12, DOI: 10.1007/s10958-017-3570-6

[15] Bandura A. I., Skaskiv O. B., Analytic functions in the unit ball of bounded L-index: asymptotic and local properties, Mat. Stud., 2017, 48(1), 37-73, DOI: 10.15330/ms.48.1.37-73 